\title{
Effectiveness of Intraoperative Laser Acupuncture Combined with Antiemetic Drugs for Prevention of Postoperative Nausea and Vomiting
}

\author{
Neslihan Unsal, MD, Zeynep Nur Akcaboy, MD,2 Ozlem Balkız Soyal, MD, \\ Erkan Yavuz Akcaboy, MD, Nevzat Mehmet Mutlu, MD, and Nermin Gogus, MD ${ }^{1}$
}

\begin{abstract}
Introduction: Postoperative nausea and vomiting (PONV) are frequent in patients undergoing laparoscopic cholecystectomy. The aim of this study is to evaluate the effectiveness of intraoperative laser acupuncture stimulation of Pericardium 6 (PC6) and Large Intestine 4 (LI4) acupoints combined with antiemetic drug prophylaxis on PONV.

Methods: A total of 88 patients, scheduled for laparoscopic cholecystectomy, were assigned into 2 groups. Group I received bilateral laser acupuncture on PC6 and LI4 acupoints after induction of anesthesia and also received antiemetic drug (metoclopramide) prophylaxis. Patients in Group II received only antiemetic drug prophylaxis. Nausea and vomiting frequencies and need for rescue antiemetic drug (ondansetron) were recorded after extubation, at 30th minute at recovery room and at 6th hour at ward.

Results: The incidence of nausea and rescue antiemetic drug need was higher at postoperative 6th hour in Group II. Vomiting was not different in groups at any time.

Conclusion: Intraoperative laser acupuncture stimulation of PC6 and LI4 acupoints combined with antiemetic drug prophylaxis decreases nausea and rescue antiemetic drug need in late postoperative period in patients undergoing laparoscopic cholecystectomy.
\end{abstract}

Keywords: laser acupuncture, antiemetic prophylaxis, postoperative nausea and vomiting, laparoscopic cholecystectomy, general anesthesia

\section{Introduction}

$\mathbf{T}$ The INCIDENCE OF POSTOPERATIVE nausea and vomiting (PONV) varies from $30 \%$ to $50 \%$ for patients undergoing surgery, which may rise up to $80 \%$ in high risk patients. ${ }^{1}$ PONV often causes patient dissatisfaction, prolonged hospital stays, unexpected readmissions, and high medical costs ${ }^{2}$ and also may cause adverse effects like bleeding, aspiration pneumonia, and electrolyte imbalance. ${ }^{3,4}$ Many antiemetic drugs are used to prevent PONV, but they have side effects and are quite expensive as well. ${ }^{5}$ Therapies that reduce PONV while avoiding side effects of drugs have the potential to increase patient satisfaction. ${ }^{6}$ This has compelled clinicians to search for nonpharmacologic interventions like acupuncture, which has previously been shown to be effective. ${ }^{7-11}$
Acupuncture has been used as a medical treatment for over 3000 years as a part of Traditional Chinese Medicine. It can be administered by needles alone, by electrical or heat stimulation through needles, and by acupressure or laser beam stimulation on acupoints along meridians. ${ }^{12,13}$ Laser acupuncture is a painless way of acupuncture, especially used in pediatric patients and the patients who have a fear of needling. Its use is very practical, painless, and devoid of major side effects. The noninvasive acupuncture technique may be more acceptable for anesthesiologists and patients. ${ }^{14}$

Pericardium 6 (PC6) acupoint has been shown to have beneficial effects on reducing PONV. ${ }^{14}$ Also in a metaanalysis it was shown that stimulation of PC6 combined with other acupoints, such as Large Intestine 4 (LI4), Stomach 36 (St36), and Large Intestine (LI11), significantly

\footnotetext{
${ }^{1}$ Anesthesiology and Reanimation Department, Saglık Bilimleri University Ankara Numune Hospital, Ankara, Turkey.

${ }^{2}$ Anesthesiology and Reanimation Department, Kırıkkale University Faculty of Medicine, Kırıkkale, Turkey.
} 
reduced the incidence of PONV compared to control group at postoperative $24 \mathrm{~h}^{15}$ The mechanism of acupuncture on prevention of PONV has not been well established, ${ }^{16}$ but it possibly reduces PONV by releasing beta-endorphins in central nervous system and/or by changes in serotonin transmission. $^{14}$

Studies concerning the timing of acupuncture in anesthetized patients have no clear evidence. ${ }^{5,15,17}$ Although administering acupuncture before induction of anesthesia has been found useful in some of the studies, there are other studies that showed no difference in PONV among preoperative, intraoperative, and postoperative application of acupuncture. ${ }^{15}$

The results of the updated Cochrane review ${ }^{18}$ for the effectiveness of PC6 acupoint stimulation in prevention of PONV showed that there is inconclusive evidence supporting the use of a combined strategy of PC6 acupoint stimulation with antiemetic drug prophylaxis, and further, high quality trials are needed for this purpose.
In this study, their aim was to evaluate the effectiveness of intraoperative stimulation of the PC6 and LI4 acupoints with laser acupuncture combined with antiemetic prophylaxis on PONV in patients undergoing laparoscopic cholecystectomy.

\section{Methods}

After the approval of Ankara Numune Research and Training Hospital Ethics Committee (approval No. E-181795), 100 patients, who will undergo laparoscopic cholecystectomy surgery, aged 18-65 years, and ASA status in preanesthetic evaluation as I or II, were recruited in this randomized, prospective, and double blinded study. All the patients were informed about the details of the study, and written informed consent was taken from all patients. But they were not informed about the group they would be in. Three patients declined to participate, and five patients were

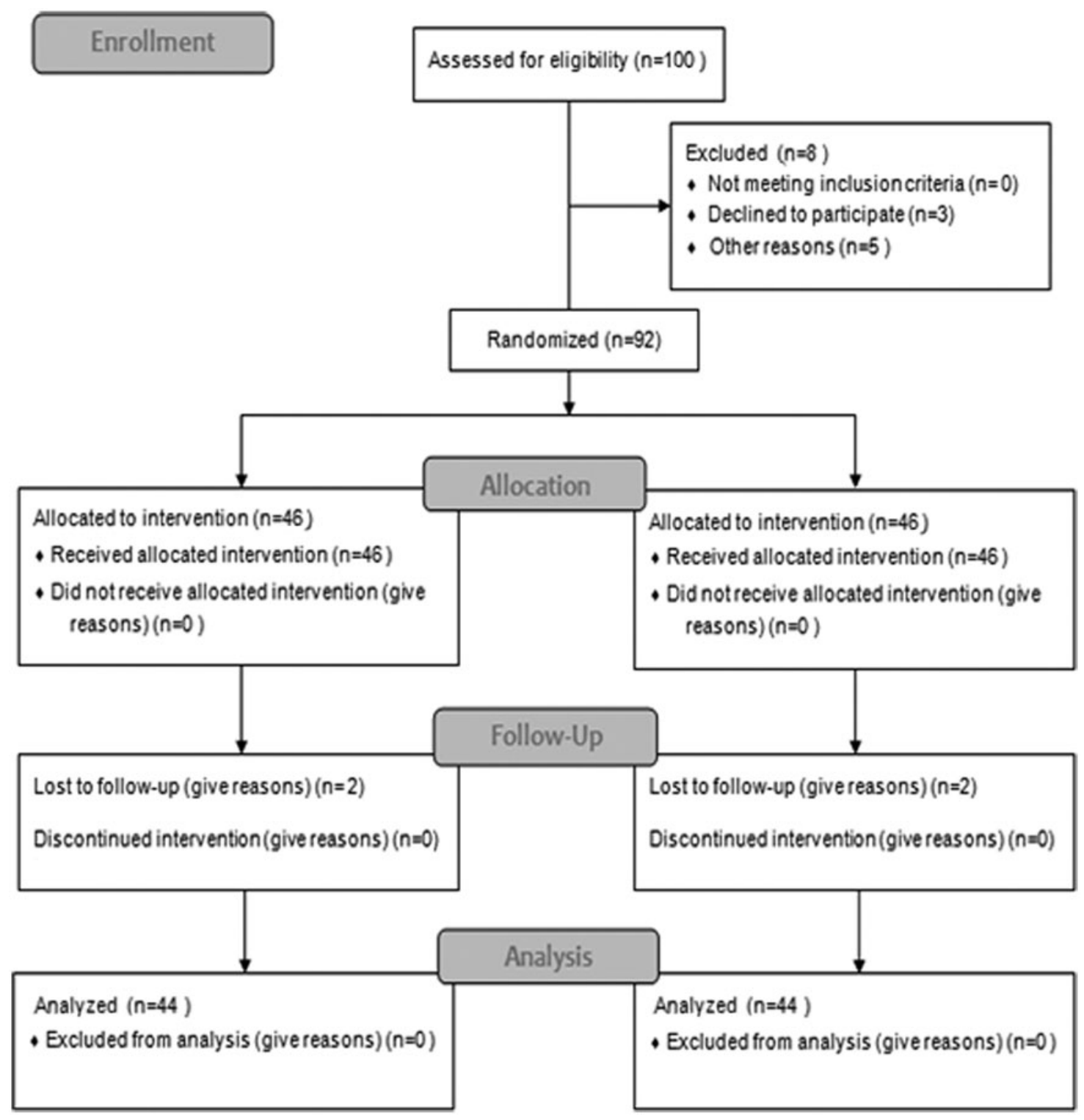

FIG. 1. CONSORT flow diagram. 
using antiemetics and excluded from the study. Ninety-two patients were randomized in two groups and allocated to intervention. But four patients were lost in follow-up because of having self-medication before their evaluation at ward. A total of 88 patients, 44 in each group, were analyzed (CONSORT flow diagram is given as Fig. 1). Patient allocation in two groups was randomized using closed envelope randomization method. In Group I, patients received antiemetic drug prophylaxis combined with laser acupuncture on PC6 and LI4 acupoints bilaterally, and in Group II, patients received antiemetic drug prophylaxis only.

In all subjects, anesthesia was induced with $2 \mathrm{mg} \cdot \mathrm{kg}^{-1}$ propofol and $0.6 \mathrm{mg} \cdot \mathrm{kg}^{-1}$ rocuronium, and tracheal intubation was facilitated afterward. Anesthesia maintenance was provided by $5 \%-6 \%$ desflurane inhalation and $0.25 \mu \mathrm{g} \cdot \mathrm{kg}^{-1} \cdot \mathrm{min}^{-1}$ remifentanil infusions. Laser acupuncture was applied to PC6 and LI4 acupoints bilaterally with the device SedatelecPremio 32 (wavelength $904 \mathrm{~nm}$, energy 2 joules per min, power output 40 W) for $30 \mathrm{sec}$ for patients in Group I. PC6 acupoint is located at the wrist between tendons of the palmaris longus and flexor carpi radialis, two cuns (one cun is equivalent to the width of the patient's thumb across the interphalangeal joint) proximal from the distal palmar crease. LI4 acupoint is located at the point halfway on the line connecting the middle of the first metacarpal bone with the middle and second metacarpal bone, when the thumb is abducted. ${ }^{15}$ Laser acupuncture device was set to "off" position, and the same points were just touched for $30 \mathrm{sec}$ in Group II.

After the surgical removal of gallbladder, metoclopramide $10 \mathrm{mg}$ and tramadol $75 \mathrm{mg}$ were administered to all patients for PONV and pain prophylaxis, respectively. At the end of the surgery, residual neuromuscular block was antagonized with neostigmine $0.03 \mathrm{mg} \cdot \mathrm{kg}^{-1}$ and atropine $0.01 \mathrm{mg} \cdot \mathrm{kg}^{-1}$. Before transferring the patient to recovery room, presence of nausea and vomiting and visual analog scale (VAS) for pain were recorded. Nausea and vomiting frequency, rescue antiemetic need, and VAS scores were recorded at postoperative 30th minute in recovery room (as early) and 6th hour at ward (as late). Rescue antiemetic need was scored as $0=$ none, $1=$ mild, $2=$ moderate, and $3=$ severe, and $4 \mathrm{mg}$ intravenous ondansetron was administered when the patient score was 3 .

To provide blindness, patients were not aware in which group they were allocated. Also treatment with laser acupuncture in Group I and only the touching of laser acupuncture device in Group II were performed after the patients were anesthetized. The same anesthesiologist, who is also an acupuncturist and experienced in acupuncture, performed all the acupuncture applications. All patients were assessed in the operating room, recovery room, and ward by different independent observers who didn't know the group allocation.

Their study aimed to test the hypothesis that combination of intraoperative laser acustimulation of the points PC6 and LI4 with antiemetic drug prophylaxis would result in reduction of anesthesia related PONV frequency compared to antiemetic drug prophylaxis alone.

Statistical analysis was performed by SPSS 24.0 program. Numerical data were analyzed with independent samples $t$ test and categorical data with Chi-square, Fisher's exact tests. Comparisons within groups were performed by Wilcoxon signed rank test. The value $p<0.05$ was regarded as
Table 1. Patient Characteristics

(Mean Standard Deviation or Median) in Groups

\begin{tabular}{lccc}
\hline & $\begin{array}{c}\text { Group I } \\
(\mathrm{n}=44)\end{array}$ & $\begin{array}{c}\text { Group II } \\
(\mathrm{n}=44)\end{array}$ & $\mathrm{p}^{*}$ \\
\hline Age (years) & $44 \pm 12.4$ & $44.6 \pm 11.8$ & 0.875 \\
Sex (M/F) & $9 / 35$ & $8 / 36$ & 1.000 \\
ASA (I/II) & $8 / 36$ & $10 / 34$ & 0.792 \\
\hline
\end{tabular}

${ }^{*} p$-Value for comparison between groups, chi square.

statistically significant. Sample size of 44 for each group was calculated as having the power of $\alpha=0.05$ and $\beta=0.90$ (effect size $d=0.7$ ).

\section{Results}

There was no significant difference between groups in age, sex distribution, and ASA status (Table 1).

The incidence of nausea after extubation and at early postoperative period (at postoperative 30th minute) was similar in both groups and not statistically different. But at late postoperative period (at postoperative 6th hour) there was no patient having nausea at Group I, although eight patients had in Group II. And this result is statistically significant between groups $(p=0.006)$.

The authors also evaluate the timecourse change of nausea incidence in each group, and this change was significantly different in Group I $(p=0.002)$, but not different in Group II $(p=0.771)$.

In group evaluation, nausea incidence at 30th min and 6th hour was not different in Group II but was different in Group I ( $p=0.771$ and $p=0.002$, respectively) (Table 2).

Vomiting was seen only in $1(2.3 \%)$ patient in Group I and in $3(6.6 \%)$ patients in Group II at postoperative 30th min, and this was not statistically different $(p=0.610)$. No vomiting was seen in either group at postoperative 6 th hour.

Seven patients in Group I and four patients in Group II needed rescue antiemetic at early postoperative period (at postoperative 30th minute), which was not statistically different between groups $(p=0.519)$. But at late postoperative period (postoperative 6th hour), no patient needed rescue antiemetics in Group I, despite eight patients needed in Group II. And this difference was found to be statistically significant ( $p=0.001)$.

VAS scores were not statistically different at any time in groups.

\section{Discussion}

PONV is a frequent complication, and there are a lot of factors influencing it. ${ }^{14,17}$ Many studies have shown that

Table 2. Nausea Incidence $[N(\%)]$ in Groups

\begin{tabular}{lllc}
\hline & $\begin{array}{c}\text { Group I } \\
(\mathrm{n}=44)\end{array}$ & $\begin{array}{c}\text { Group II } \\
(\mathrm{n}=44)\end{array}$ & $\begin{array}{c}\text { Between } \\
\text { groups } \mathrm{p}^{*}\end{array}$ \\
\hline After extubation & $0(0)$ & $2(4.5)$ & 0.494 \\
Postoperative 30th minute & $9(20.5)$ & $6(13.6)$ & 0.571 \\
Postoperative 6th hour & $\mathbf{0 ( 0 ) * *}$ & $8(18.2)$ & 0.006 \\
In groups $p^{* *}$ & $\mathbf{0 . 0 0 2}$ & 0.771 & \\
\hline
\end{tabular}

$* p$-Value for comparison between groups, chi square.

$* * p$-Value for comparison in groups, chi square.

Bold values indicate statistical significance. 
drugs such as metoclopramide, dexamethasone, and ondansetron can reduce PONV. Although potency of these drugs is different, they have a limited effect on PONV and they also have adverse effects such as sedation, headache, and extrapyramidal side effects in addition to high costs. ${ }^{19,20}$

Acupuncture is a nonpharmacologic alternative intervention for the prevention of PONV and has the benefit of lower costs. The antiemetic effects of acupuncture may be attributable to changes in the activity of neurochemicals. When an acupoint is stimulated, low frequency electrical stimulus is generated, which activates the A- $\beta$ and $A-\delta$ fibers, thus releasing endorphins in the central nervous system, which ultimately strengthens the intrinsic antivomiting pathway. It appears that a change occurs in the serotonin transmission pathway, resulting in antivomiting effects. The neurochemicals released by acupoint stimulation desensitize the vomiting center in the brain, resulting in the prevention of nausea and vomiting. ${ }^{21}$

Any therapy that reduces PONV while avoiding the unwanted side effects of routinely used drugs has the potential to increase patient satisfaction and also has important economic implications for the hospital.

Acupuncture of PC6 in patients has been shown to reduce the incidence of PONV. ${ }^{22}$ The clinical effects of acupoint PC6 are digestive effects, including mitigation of nausea and vomiting. $^{23,24}$ The updated Cochrane review ${ }^{18}$ about PC6 acustimulation in prevention of PONV concluded that the effects of PC6 within multimodal prophylaxis of PONV (additive or synergistic effects of PC6 acupuncture on antiemetic use) and also the combined usage and effects of PC6 and other acupoints remain incompletely understood and require further investigations. In their study the authors primarily aimed to compare the additive effect of acupuncture when combined with antiemetic drug prophylaxis. The authors also aimed to evaluate the adjunctive effect of LI4 acustimulation, so that both PC6 and LI4 acupoints were used bilaterally in acupuncture applied patients. LI4 is a commonly used acupoint in pain management. ${ }^{25}$ But also its adjunctive effects on PONV when combined with PC6 were studied and reported as effective. ${ }^{26}$ In their study the authors found that adding acustimulation of PC6 and LI4 acupoints reveals no difference in PONV and rescue antiemetic usage in early postoperative period but reduces them in late postoperative period. This can be explained by time related decrease in the effects of antiemetic drugs in the late postoperative period; the long-term antiemetic effect of acupuncture prevents nausea and also decreases the additional antiemetic drug usage. This can be defined as useful in terms of increasing patient satisfaction and reducing the costs.

In their study the authors preferred to use laser acupuncture because of its advantages, and all the treatments were successful without any side effects. In recent studies, the effectiveness of its usage is shown. ${ }^{14,22}$ Although laser acupuncture was applied after induction of anesthesia in their study, patients' acceptance of acupuncture treatment was easier because of its noninvasive, needleless, and painless properties. The authors think that these useful properties of laser acupuncture will be more important and preferred in studies when the acustimulation will be applied in awake patients.

Timing of acupuncture in anesthetized patients is another subject to review. Variations in timing of acupuncture were suggested as a source of heterogeneity in previous metaanalysis of acupuncture for PONV. No clear evidence exists for the optimal timing of acupuncture (pre, intra, or postoperatively). ${ }^{15,18}$ Studies at first postulated that stimulation performed before induction of anesthesia seemed to be important. ${ }^{11,27,28}$ But in recent studies favorable effects of perioperative administration of acupuncture, including the intraoperative period, were reported in some trials. ${ }^{22}$ In their study the results supported the effectiveness of intraoperative acustimulation of PC6 and LI4 points on PONV. May be it is not so important when using noninvasive, painless laser acupuncture, but especially when acupuncture is to be applied using needles, it can be more comfortable for the patients to have the treatment under anesthesia.

As a result, their study indicates that combining intraoperative laser acustimulation of PC6 and LI4 acupoints with antiemetic drug prophylaxis decreases nausea and rescue antiemetic drug need in late postoperative follow-up period in patients undergoing laparoscopic cholecystectomy. Using laser acupuncture can decrease the need for antiemetic drugs and this can be considered useful in preventing side effects of antiemetic drugs, decreasing costs, and increasing patient satisfaction. Also the painless and noninvasive properties of laser acupuncture can be the reason for preferance especially when applied in awake patients.

\section{Author Disclosure Statement}

No competing financial interests exist.

\section{Funding Information}

No funding was received for this article.

\section{References}

1. Gan TJ, Diemunsch P, Habib AS, et al. Consensus guidelines for the management of postoperative nausea and vomiting. Anesth Analg 2014;118:85-113.

2. Hill RP, Lubarsky DA, Philips-Bute B, et al. Costeffectiveness of prophylactic antiemetic therapy with ondansetron, droperidol, or placebo. Anesthesiology 2000;92: 958-967.

3. Watcha MF, White PF. Postoperative nausea and vomiting. Its etiology, treatment, and prevention. Anesthesiology 1992;77:162-184.

4. Palazzo MG, Strunin L. Anesthesia and emesis. I: Etiology. Can Anaesth Soc J 1984;31:178-187.

5. Lee S, Lee MS, Choi DH, Lee SK. Electroacupuncture on PC6 prevents opioid-induced nausea and vomiting after laparoscopic surgery. Chin J Integr Med 2013;19: 277-281.

6. Weeks EM, Trinca J, Zheng Z. Knowledge of and willingness to try acupuncture for postoperative nausea and vomiting: An Australian survey of surgical patients. Acupunct Med 2017;35:345-351.

7. Fassoulaki A, Papilas K, Sarantopoulos C, Zotou M. Transcutaneous electrical nerve stimulation reduces the incidence of vomiting after hysterectomy. Anesth Analg 1993;76:1012-1014.

8. Al-Sadi M, Newman B, Julious SA. Acupuncture in prevention of postoperative nausea and vomiting. Anesthesia 1997;52:658-661. 
9. White PF. Are nonpharmacologic techniques useful alternatives to antiemetic drugs for the prevention of nausea and vomiting?, Anesth Analg 1997;84:712-714.

10. Harmon D, Gardiner J, Harrison R, Kelly A. Acupressure and the prevention of nausea and vomiting after laparoscopy. Br J Anaesth 1999;82:387-390.

11. Fan CF, Tanhui E, Joshi S, et al. Acupressure treatment for prevention of postoperative nausea and vomiting. Anesth Analg 1997;84:821-825.

12. Baines D. Postoperative nausea and vomiting in children. Paediatr Anaesth 1996;6:7-14.

13. Henzi I, Walder B, Tramer MR. Metoclopramide in the prevention of postoperative nausea and vomiting: A quantitative systematic review of randomized, placebo controlled studies. Br J Anaesth 1999;83:761-771.

14. Butkovic D, Toljan S, Matolic M, et al. Comparison of laser acupuncture and metoclopramide in PONV prevention in children. Pediatr Anaesth 2005;15:37-40.

15. Cheong KB, Zhang J, Huang Y, Zhang Z. The effectiveness of acupuncture in prevention and treatment of postoperative nausea and vomiting-A systematic review and metaanalysis. PLoS One 2013;8:e82474.

16. Lee A, Done ML. The use of nonpharmacologic techniques to prevent postoperative nausea and vomiting: A metaanalysis. Anesth Analg 1999;88:1362-1369.

17. Kim KH, Kim DH, Bae JM, et al. Acupuncture and PC6 stimulation for the prevention of postoperative nausea and vomiting in patients undergoing elective laparoscopic resection of colorectal cancer: A study protocol for a threearm randomized pilot trial. BMJ Open 2017;7:e013457.

18. Lee A, Chan SK, Fan LT. Stimulation of the wrist acupuncture point PC6 for preventing postoperative nausea and vomiting. Cochrane Dtabase Syst Rev 2015;2:CD003281.

19. Rose JB, Martin TM, Corddry DH, et al. Ondansetron reduces the incidence and severity of strabismus repair vomiting in children. Anesth Analg 1994;79:486-489.

20. Tramer M, Moore A, McQuay H. Prevention of vomiting after paediatric strabismus surgery: A systematic review using the numbers-needed-to-treat method. Br J Anaesth 1995;75:556-561.
21. Kim SI, Yoo IS, Park HN, et al. Transcutanenous electrical stimulation of the P6 acupoint reduces postoperative nausea after minor breast surgery. Kor J Anesthesiol 2004;47:834839.

22. Schlager A, Offer T, Baldissera I. Laser stimulation of acupuncture point $\mathrm{P} 6$ reduces postoperative vomiting in children undergoing strabismus surgery. Br J Anaesth 1998; 81:529-532.

23. Wang C, Zhou DF, Shuai XW, et al. Effects and mechanism of electroacupuncture at PC6 on frequency of transient lower esophageal sphincter relaxation in cats. World $\mathbf{J}$ Gastroenrerol 2001;13:4873-4880.

24. Somri M, Vaida SJ, Sabo E, et al. Acupuncture versus ondansetron in the prevention of postoperative vomiting. A study of children undergoing dental surgery. Anaesthesia 2001;56:927-932.

25. Seevaunnamtum SP, Bhojwani K, Abdullah N. Intraoperative electroacupuncture reduces postoperative pain, analgesic requirement and prevents postoperative nausea and vomiting in gynaecological surgery: A randomized controlled trial. Anesth Pain Med 2016;6:e40106.

26. Alizadeh R, Esmaeili S, Shoar S, et al. Acupuncture in preventing postoperative nausea and vomiting: Efficacy of two acupuncture points versusu single one. J Acupunct Meridian Stud 2014;7:71-75.

27. Allen DL, Kitching AJ, Nagle C. P6 acupressure and nausea and vomiting after gynaecological surgery. Anaesth Intensive Care 1994;22:691-693.

28. Dundee JW, Ghaly RG, Bill KM, et al. Effect of stimulation of the P6 antiemetic point postoperative nausea and vomiting. Br J Anaesth 1989;63:612-618.

Address correspondence to: Zeynep Nur Akcaboy, MD Anesthesiology and Reanimation Department Kirlkkale University Faculty of Medicine 71450 Yahsihan, Kırıkkale Turkey

E-mail: zakcaboy@gmail.com 